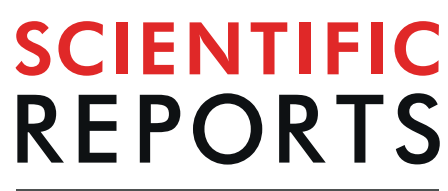

\title{
Fabrication of atomic junctions with experimental parameters optimized using ground-state searches of Ising spin computing
}

\author{
Shotaro Sakai, Yosuke Hirata, Mitsuki Ito @ \& Jun-ichi Shirakashi* \\ Feedback-controlled electromigration (FCE) is employed to control metal nanowires with quantized \\ conductance and create nanogaps and atomic junctions. In the FCE method, the experimental \\ parameters are commonly selected based on experience. However, optimization of the parameters \\ by way of tuning is intractable because of the impossibility of attempting all different combinations \\ systematically. Therefore, we propose the use of the Ising spin model to optimize the FCE parameters, \\ because this approach can search for a global optimum in a multidimensional solution space within \\ a short calculation time. The FCE parameters were determined by using the energy convergence \\ properties of the Ising spin model. We tested these parameters in actual FCE experiments, and \\ we demonstrated that the Ising spin model could improve the controllability of the quantized \\ conductance in atomic junctions. This result implies that the proposed method is an effective tool for \\ the optimization of the FCE process in which an intelligent machine can conduct the research instead of \\ humans.
}

Electromigration (EM) has been known to degrade the structures and interconnects of integrated circuits. In recent years, however, EM has become a topic of intense interest because of its potential for fabricating nanoscale structures. Most of this work involved the investigation of EM on the nanoscale with the objective of utilizing it for the fabrication of nanogaps for single-electron transistors ${ }^{1,2}$, single-molecule transistors ${ }^{3,4}$, and atomic junctions for nanoscale switches ${ }^{5-7}$. EM is strongly related to the current density and temperature of the metallic conductor $^{8}$. Typical electromigrated nanogaps formed with a single voltage ramp tend to exhibit extremely high tunnel resistances and then catastrophically break ${ }^{9}$. In addition, high local temperatures can be reached during $\mathrm{EM}^{10}$, which can cause large gap openings and residual metallic nanoparticles ${ }^{11}$. Recently, feedback-controlled electromigration (FCE) methods have been developed to enable temperature control ${ }^{12}$. The FCE process actively tunes the applied voltage in response to the changing resistance/conductance of the metal nanowires. The FCE scheme has been successfully employed to create nanogaps safely and reliably by controlling the quantum states of conductance in atomic junctions ${ }^{13-15}$. However, the parameters that are used in actual experimental procedures are often selected from published reports or adjusted subsequently by a trial-and-error approach, which is not only time-consuming but also highly dependent on experience. Furthermore, the scheme rarely fully utilizes the correlations between parameters because of the substantial amount of data.

Recently, the Ising spin model, which is a statistical mechanics model of magnetism invented by Wilhelm Lenz in $1920^{16}$, has garnered considerable interest as a potential new computing architecture. The Ising spin model can be applied to many combinatorial optimization problems ${ }^{17}$, and it can determine the optimal solution from large numbers of candidate solutions within short calculation times ${ }^{18}$. The combinatorial optimization problems are mapped to find the ground state of the corresponding Ising spin model. In recent years, hardware implementations analogous to the Ising spin model have also been proposed ${ }^{18-22}$. They have received considerable interest as a potential new computing paradigm for addressing a wide range of social issues. In fact, many research studies have already been reported ${ }^{23-30}$. Here, we show that Ising spin computing can be used for FCE parameter optimization by directly mapping the parameter selection, which is a "combinatorial optimization problem," onto the Ising Hamiltonian. In other words, we use Ising spin computing for parameter optimization based on machine 


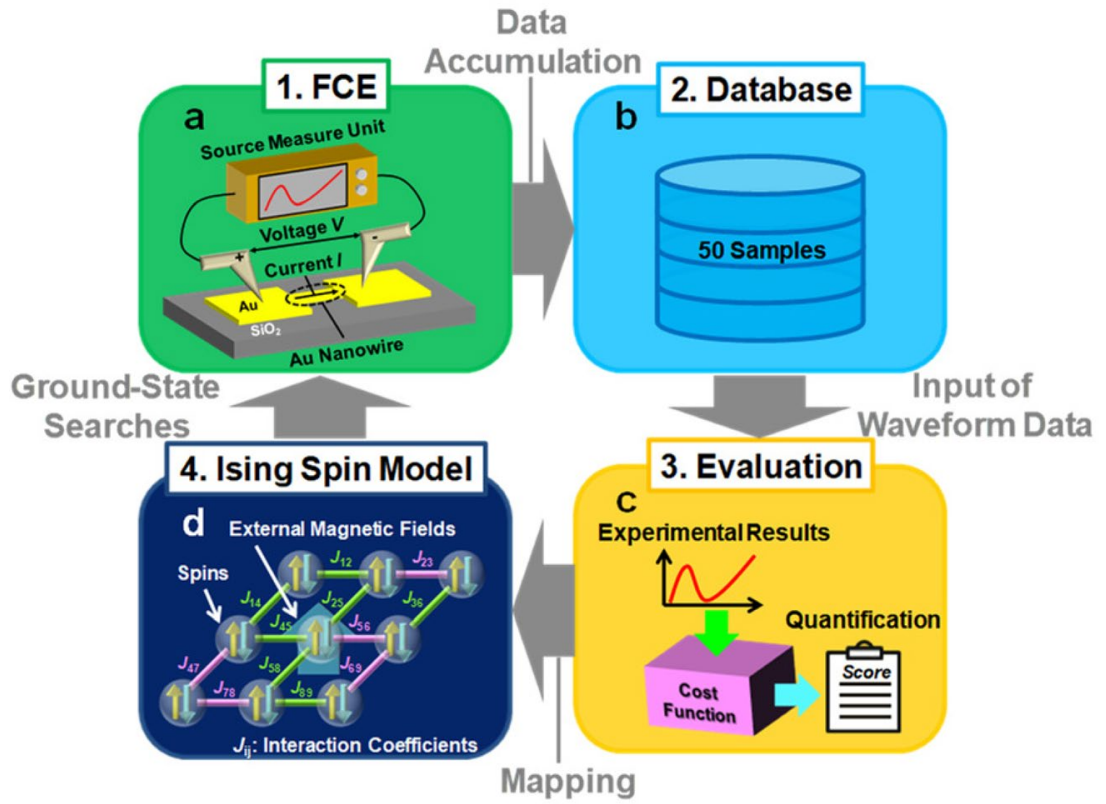

$\mathbf{e}$

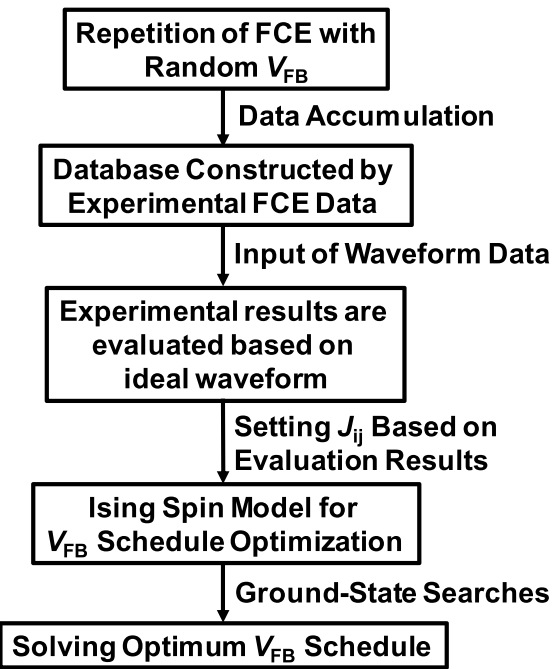

Figure 1. System flow for optimization of $V_{\mathrm{FB}}$ schedule. (a) Process of FCE with random $V_{\mathrm{FB}}$ values, with subsequent storage of the experimental data in a database. (b) Evaluation of experimental data by the defined cost function. (c) Mapping of the $V_{\mathrm{FB}}$ schedule onto the Ising spin model. (d) Optimization of $V_{\mathrm{FB}}$ schedule via ground-state searches of the Ising spin model, and application of the schedule to the FCE experiments. (e) Block diagram of system flow.

learning beyond the baseline model that finds the ground state. Our work differs from that presented in previous reports in that we apply the optimization results obtained with the Ising spin computing to actual experiments in the research domain. The ability to optimize FCE parameters using Ising spin computing is equivalent to being able to address this problem with quantum annealing machines/quantum annealers based on the Ising spin model. Compared with the method of simulated annealing ${ }^{31}$, the performance of quantum annealers is superior and advantageous for solving problems with energy landscapes that have complicated tunneling barriers ${ }^{32}$. Therefore, in the future, complex optimization problems concerned with physical phenomena are also foreseen to be solved efficiently using quantum annealers. Our approach opens new avenues for bridging the gap between key problems of parameter optimization in fabricating nanoscale devices and their implementation to Ising spin computing.

Figure 1 schematically illustrates the proposed system. We first outline our approach for constructing a database of the FCE (Fig. 1a) and then show how it is "informed" from our database (Fig. 1b). In this study, we focused on the feedback (FB) voltage $V_{\mathrm{FB}}$, which is one of the FB parameters, and collected the data of $V_{\mathrm{FB}}$. $V_{\mathrm{FB}}$ determines the amount of voltage reduction, which suppresses the heat of the channel. Therefore, this parameter plays an important role for the control of quantized conductance. In the mapping step (Fig. 1c), the problem of optimizing $V_{\mathrm{FB}}$ is mapped into the Ising spin model. The mapping step consists of two parts. One represents the 
solution of the original problem by the combination of binary spins and the other determines the values of the interaction coefficients and external magnetic coefficients such that the spin values at the ground state correspond to the solution of the original problem. Finally, we applied an optimum $V_{\mathrm{FB}}$ schedule obtained by the ground-state searches of the Ising spin model to the FCE experiments to confirm the usefulness of our system (Fig. 1d). In summary, Fig. 1e outlines the flow of the experiment.

\section{Results and Discussion}

Accumulation of FCE experimental data into database. Conventional electron-beam lithography and lift-off processes were used to fabricate Au nanowires with a constricted channel structure. First, Au nanowires were patterned on resist-coated $\mathrm{SiO}_{2} / \mathrm{Si}$ substrates using electron-beam lithography. Electron-beam evaporation of $\mathrm{Ti}(5 \mathrm{~nm})$ and $\mathrm{Au}(20 \mathrm{~nm})$ was then carried out using a developed resist pattern as a template. Finally, the unnecessary metal and resist layers were removed.

The detailed experimental steps of the FCE procedures are as follows. First, the applied voltage $V$ was automatically increased with controlled loop rates in voltage steps $V_{\text {STEP }}(=1.5 \mathrm{mV})$ while the current $I$ was monitored until EM occurred. Next, when the differential conductance of the Au nanowires reached the threshold differential conductance $G_{\mathrm{TH}}(=20 \mathrm{mS}), V_{\mathrm{FB}}$ automatically reduced the applied voltage $V$, in which the voltage was reduced by $10-90 \%$ of the existing applied voltage value to slow down the EM-induced breaking process. Note that the differential conductance is obtained between measurement points and compared with $G_{\mathrm{TH}}$ during the FCE process. Finally, the applied voltage $V$ was ramped up again for the next EM cycle. In this procedure, we designed the FCE schedules with randomly varying values of $V_{\mathrm{FB}}$. These schedules were applied to 50 Au nanowires, and the FCE data were stored in a database.

Evaluation of FCE experimental parameters using cost function. We set the cost function, which evaluates the values of $V_{\mathrm{FB}}$, to map the $V_{\mathrm{FB}}$ schedule onto the Ising spin model. Here, the cost function is assumed to become large when the quantized conductance smoothly plateaus, and the step-wise conductance subsequently and clearly drops by $G_{0}\left(G_{0}=2 e^{2} / h=77.6 \mu \mathrm{S}\right.$, where $e$ is the electron charge, and $h$ is Planck's constant $)$. The time-dependent conductance curve corresponds to the change in the structure at the constricted section of the Au nanowires ${ }^{33}$. Therefore, we set five variables to represent the features of the conductance curve. Figure $2 \mathrm{a}$ shows a schematic of the conductance trace and applied voltage $V$ and represents the variables in the cost function. $P_{1}$ is the number of data points counted from $G_{\text {ref }}$ in the quantized conductance that lie within a conductance window $G_{\text {ref }} \pm 0.5 G_{0}$ in each conductance trace, $L$ is the number of data points from $G_{\text {ref }}$ to the end of the FB cycle $G_{\mathrm{E}}, D$ is the decrease in conductance in the region of EM occurrence, $F$ is the decrease in conductance during one EM cycle between the start of the FB cycle $G_{\mathrm{S}}$ and $G_{\mathrm{E}}$, and $P_{2}$ is the change in conductance between $G_{\min }$ and $G_{\max }$ during the application of ramp voltage. Furthermore, $N$ is set as the number of voltage FB cycles during the FCE process. In an ideal control scheme, $D$ and $F$ are close to $1 G_{0}, P_{1} / L$, which means the stability of the conductance trace, is set to 1 , and $P_{2}$ is set to $0 G_{0}$. Therefore, the cost function has the form

$$
\text { Score }_{V \mathrm{FB}} \equiv s_{n}(X)=\frac{P_{1} / L}{|D-1|+|F-1|+\left|P_{2}\right|} .
$$

Here, we defined the output using Eq. (1) as the Score of $V_{\mathrm{FB}}$ values $(X \%)$ at $n$th times of FB. When the conductance trace is close to these ideal values, the $s_{n}(X)$ increases until infinity. Here, Eq. (1) is applied below 40 $G_{0}$. In this region, the electron transport is in the ballistic regime and the EM is activated with the one-by-one removal of atoms, which is driven by the kinetic energy transfer from single conduction electrons to single Au atoms ${ }^{34,35}$. Hence, using Eq. (1), we can evaluate the $V_{\mathrm{FB}}$ values from the point of view of the conductance behavior under the voltage $\mathrm{FB}$ process.

Figure $2 \mathrm{~b}$ presents the typical result of the conductance $G$ of the Au nanowires and the voltage $V$ as a function of the process time $t$ when a random $V_{\mathrm{FB}}$ schedule is applied to an FCE. The vertical axis of Fig. $2 \mathrm{~b}$ is the conductance $G$ of the sample over $G_{0}$. Furthermore, it should be noted that the decrease in the conductance of " 1 " $G_{0}$ corresponds to a one-by-one removal of $\mathrm{Au}$ atoms ${ }^{34,35}$. Three FB points are indicated by black arrows, and Fig. $2 \mathrm{~b}$ also presents the results of $s_{n}(X)$ obtained from Eq. (1). From Fig. $2 \mathrm{~b}$, the $s_{11}(40)$, in which $V_{\mathrm{FB}}=40 \%$ at FB point 2 , indicating smooth plateaus caused by quantized conductance, is much higher than $s_{10}(20)$ (at FB point 1 ) and $s_{12}(50)$ (at FB point 3). Therefore, the controllability of the quantized conductance can be evaluated with Eq. (1).

We quantify the correlation between the nearest-neighbor $V_{\mathrm{FB}}$ values through the following formula:

$$
\text { Score }_{\text {trans }} \equiv s_{n, n+1}(X, Y)=\frac{s_{n}(X)+s_{n+1}(Y)}{2},
$$

where the combination of $s_{n}(X)$ and $s_{n+1}(Y)$ represent $s_{n, n+1}(X, Y)$ occurring at $V_{\mathrm{FB}}$ of $X \%$ and $Y \%$ during an FCE process. In Fig. 2c, we show the mutual information between $V_{\mathrm{FB}}$ values. For example, $s_{1,2}(10,20)$ represents the evaluation for $V_{\mathrm{FB}}$ transition from $10 \%$ to $20 \%$ at the first to the second voltage FB cycles. In the FCE method, generally, Au nanowires should gradually narrow with the progress of FB cycles/steps, which is due to the EM. In other words, the conductance of Au nanowires should decrease with the evolution of FB processes. Here we hypothesize that a correlation exists between subsequent feedback steps because the EM proceeds by removing one atom at a time. Therefore, the correlation between nearest-neighbor $V_{\mathrm{FB}}$ values is assumed to be equivalent to considering the junction states between FB cycles. The pixels in Fig. 2c illustrate the fact that the transitions of the $V_{\mathrm{FB}}$ values strongly influence the evaluated values of an FCE process. As a result, we obtain the suitable combination of $V_{\mathrm{FB}}$ values for the controllability of quantized conductance during the FCE. 
a

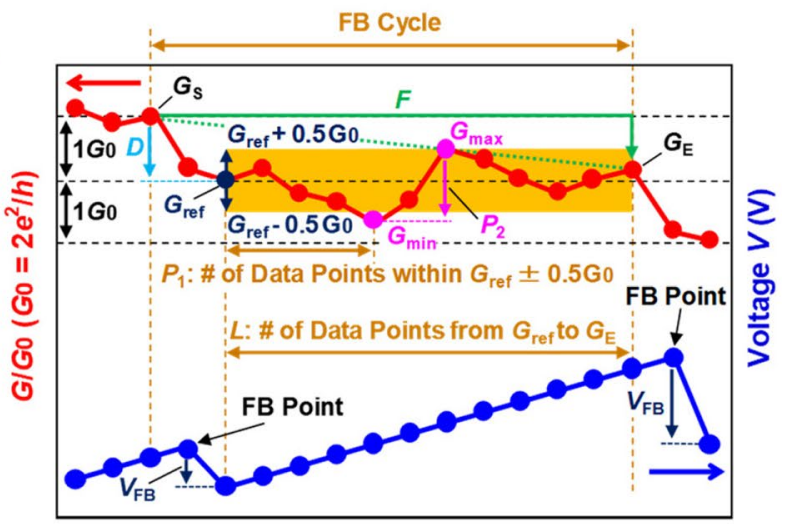

Process Time $t$ (s)

b

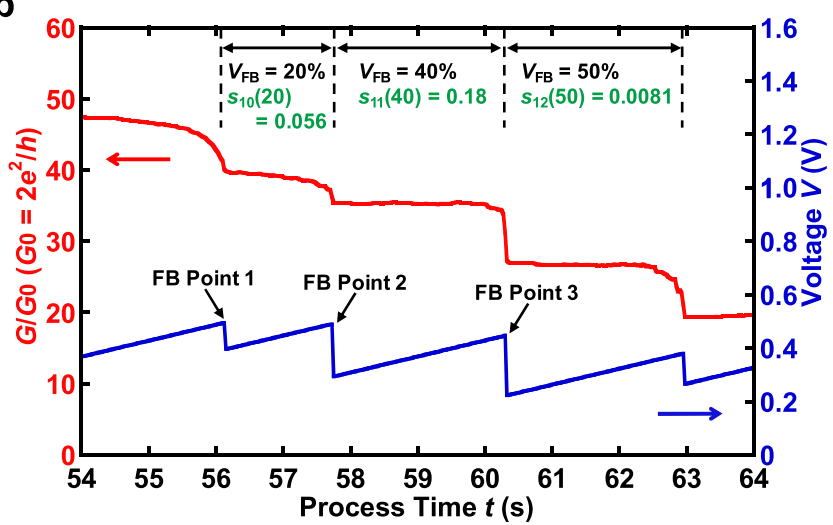

C

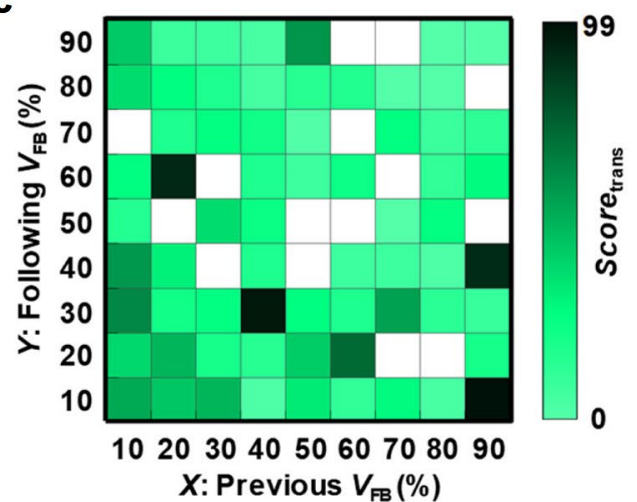

Figure 2. Construction of database and evaluation of $V_{\mathrm{FB}}$. (a) Schematic of conductance trace and each variable for cost function, where $D=G_{\mathrm{S}}-G_{\mathrm{ref}}, P_{1}$ is the number of data points that meet the condition of $\mid G-$ $G_{\text {ref }} \mid<0.5 G_{0}, P_{2}=G_{\max }-G_{\min }, F=G_{\mathrm{S}}-G_{\mathrm{E}}$, and $L$ is the number of data points from $G_{\text {ref }}$ to $G_{\mathrm{E}}$. (b) Typical time evolutions of the junction conductance, $G / G_{0}(\mathrm{red})$, where $G_{0}=2 e^{2} / h$, and the junction voltage, $V$ (blue), with random $V_{\mathrm{FB}}$ values. The evaluation is from the viewpoint of controllability of the quantized conductance using Eq. (1) at three FB points between 54 and 64 s. A larger Score $_{V F B}$, which is infinite in an ideal case, indicates a higher evaluation. FB point 2 indicates that the quantized conductance smoothly plateaus, and Eq. (1) gives the high Score $_{\mathrm{VFB}}$. (c) Interaction information between pairs of $V_{\mathrm{FB}}$. Highly and lowly scored transitions appear as dark and bright pixels, respectively. In addition, an empty pixel represents no information in the database. Each value shown is linearly scaled to fall within the range of $[0,99]$.

Mapping of FCE schedules onto Ising spin model. We map the $V_{\mathrm{FB}}$ schedule onto the Ising spin model using the cost function described above. The formulation of $H_{\text {Ising, }}$, the system Hamiltonian (total energy of the system), for the Ising spin model can be described by

$$
H_{\text {Ising }}=-\sum_{i} \sum_{x} \sum_{j} \sum_{y} J_{i x, j y} \sigma_{i, x} \sigma_{j, y}-\sum_{i} \sum_{x} h_{i, x} \sigma_{i, x} .
$$


Typically, a real-world problem formulation requires conversion to the Ising Hamiltonian. In this research, the $V_{\mathrm{FB}}$ schedule is mapped into fully connected two-dimensional Ising spin cells consisting of $N$ (row) $\times 9$ (column) spins. As a result, the minimum value of the system energy and the corresponding optimum provide the solution to the original problem. In this model, each row corresponds to the order of $V_{\mathrm{FB}}$ values $(i, j \in 1, \ldots, N)$ and each column corresponds to the $V_{\mathrm{FB}}$ values $(x, y \in 1, \ldots, 9)$. Each spin $\left(\sigma_{i, x}\right.$ or $\left.\sigma_{j, y}\right)$ takes a binary value of " +1 " or " 0 ," which represents an up spin or a down spin. Furthermore, a mapped $V_{\mathrm{FB}}$ schedule is finally represented by a trace of spin value " +1 ." The strength of the connection between spin $(i, x)$ and spin $(j, y)$ is denoted by $J_{i x, j y}$. Each spin is also subject to the external magnetic field of $h_{i, x}$. The formulation of the Ising Hamiltonian for the optimization of the FCE process is explained below. First, to select the highly rated schedules, the term

$$
H_{\text {evaluation }}=\sum_{i} \sum_{x} \sum_{j} \sum_{y} \delta_{i, j-1} W_{x, y} \sigma_{i, x} \sigma_{j, y}
$$

is set into the system Hamiltonian, where $W_{x, y}$ is based on Eq. (2). For example, $W_{1,2}$ is decided based on the evaluation of the transition of $V_{\mathrm{FB}}$ from $10 \%$ to $20 \%$. In addition, $\delta_{i, j}$ is equal to $1(i=j)$ or 0 (otherwise). Penalty terms are added such that spin states that do not correspond to valid solutions are penalized. Then, to ensure no two $V_{\mathrm{FB}}$ overlap, we introduce a penalty term,

$$
H_{\text {overlap }}=\sum_{i} \sum_{x} \sum_{j} \sum_{y} \delta_{i, j} \sigma_{i, x} \sigma_{j, y} .
$$

Finally, to ensure that the total number of spins on " +1 " is exactly $N$,

$$
H_{\mathrm{total}}=\sum_{i} \sum_{x} \sum_{j} \sum_{y} \delta_{i, j} \delta_{x, y} \sigma_{i, x} \sigma_{j, y}
$$

is added to the system Hamiltonian. Hence, the cost function for $V_{\mathrm{FB}}$ scheduling, which is represented by $H_{\mathrm{FCE}}$, is given as

$$
H_{\mathrm{FCE}}=\frac{A}{2} H_{\text {evaluation }}+\frac{B}{2} H_{\text {overlap }}+\frac{C}{2} H_{\text {total }}-D \sum_{i} \sum_{x} h_{i, x} \sigma_{i, x},
$$

where $A, B, C$, and $D$ are positive constants. A valid solution will have $H_{\text {overlap }}=H_{\text {total }}=0$.

Solution searches of FCE experimental parameters using Ising spin computing. Here, we first provide and set the optimal solution for our simulation to measure the performances of our Ising spin computing. According to the database, which consists of 50 samples, the average number of FB steps in the FCE process in a sample was approximately ten. Therefore, in the calculation experiments, we set 90 spins $(N=10, N \times 9=90)$ with 10,000 iterative cycles. A single iterative cycle describes the flips of all spins chosen at random. Furthermore, the update of spins depends on the temperature $T$ through the simulated annealing procedure ${ }^{31}$ with the aim of escaping from a local minimum. In each step of this algorithm, a spin is given a small random displacement and the resulting change, $\Delta H$, in the energy of the system is computed. The probability that the configuration is accepted is $P(\Delta H)=\exp (-\Delta H / T)$. Random numbers uniformly distributed in the interval $(0,1)$ are selected and compared to $P(\Delta H)$. If they are less than $P(\Delta H)$, the new configuration is retained; if not, the original configuration is used to start the iteration.

In the test problem we set, at the global minimum with a system energy $H_{\mathrm{FCE}}$ of $-4,175$, the final spin status appears and traces the following: $V_{\mathrm{FB}}(\%)=60 \rightarrow 70 \rightarrow 80 \rightarrow 90 \rightarrow 10 \rightarrow 20 \rightarrow 30 \rightarrow 40 \rightarrow 50 \rightarrow 60$. Figure 3 a shows the system energy $H_{\mathrm{FCE}}$ and temperature $T$ as a function of the number of iterative cycles. In this case, the computation is complete within seconds because we used conventional computing resources. Over time the energy decreases and is characterized by upward and downward movements. After 10,000 iterative cycles, the system energy reaches $-4,175$. Therefore, the system energy is considered to have reached the global minimum we set through the ground-state searches of the Ising spin computing. Figure $3 \mathrm{c}$ also presents the final spin status of the system at the global minimum energy. Each spin is expressed by a square. The spin state in orange in Fig. $3 \mathrm{c}$ represents the optimal solution set of $V_{\mathrm{FB}}$ scheduling with $H_{\mathrm{FCE}}=-4,175$. Based on these results, Ising spin computing can determine the optimal solution of experimental parameters for the $V_{\mathrm{FB}}$ schedule.

To demonstrate the utility of our Ising spin computing, we apply it to a database containing conductance measurements of $50 \mathrm{Au}$ nanowires. Figure $3 \mathrm{~b}$ plots the process of the ground-state search based on the experimental data stored in the database. As seen in Fig. 3b, the energy profiles exhibit similar characteristics to those in Fig. 3a. Furthermore, the final ground-state spin configuration in Fig. 3d represents a suitable $V_{\mathrm{FB}}$ schedule that alternates between $V_{\mathrm{FB}}$ of $20 \%$ and $60 \%$ with time. This result traces the spins with highly evaluated values of $V_{\mathrm{FB}}$ obtained from the database consisting of 50 samples. Thus, Ising spin computing progressed from the entirely random data of the $V_{\mathrm{FB}}$ schedule towards an indication of the experimental parameters for the $V_{\mathrm{FB}}$ schedule. Clearly, an ordered $V_{\mathrm{FB}}$ schedule can be generated from the disordered data stored in the database by using the proposed Ising spin computing model.

Score of $V_{\mathrm{FB}}$ schedules obtained with solution searches. Next, we investigated the ability of our Ising spin computing to search for the ground-state solution. We did this by comparing the score of the $V_{\mathrm{FB}}$ scheduling obtained from the Ising spin computing and random selection. Here, we defined the Score of $V_{\mathrm{FB}}$ schedule as 
a

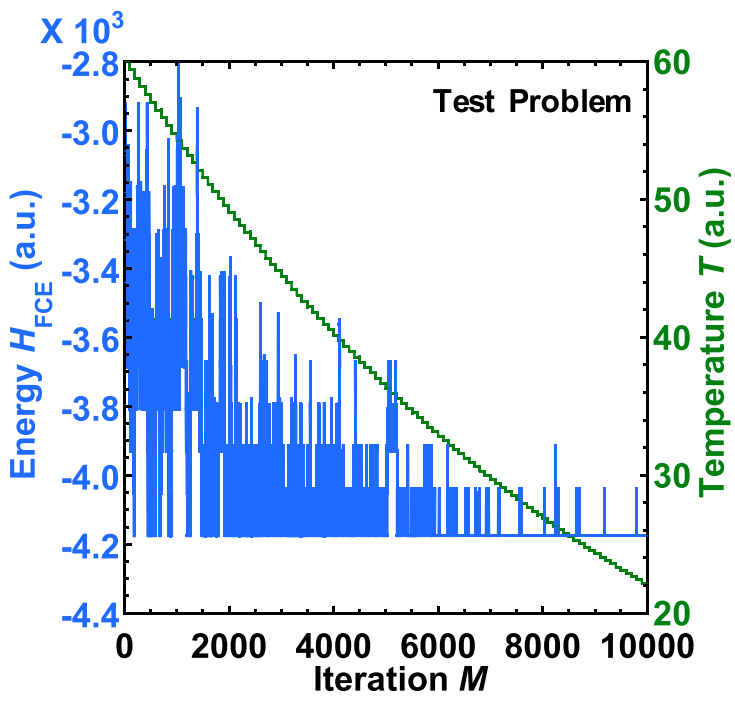

C

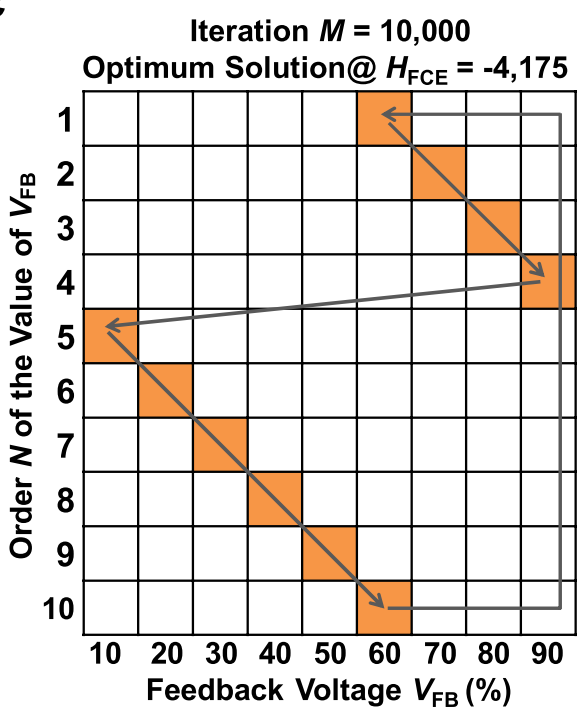

d

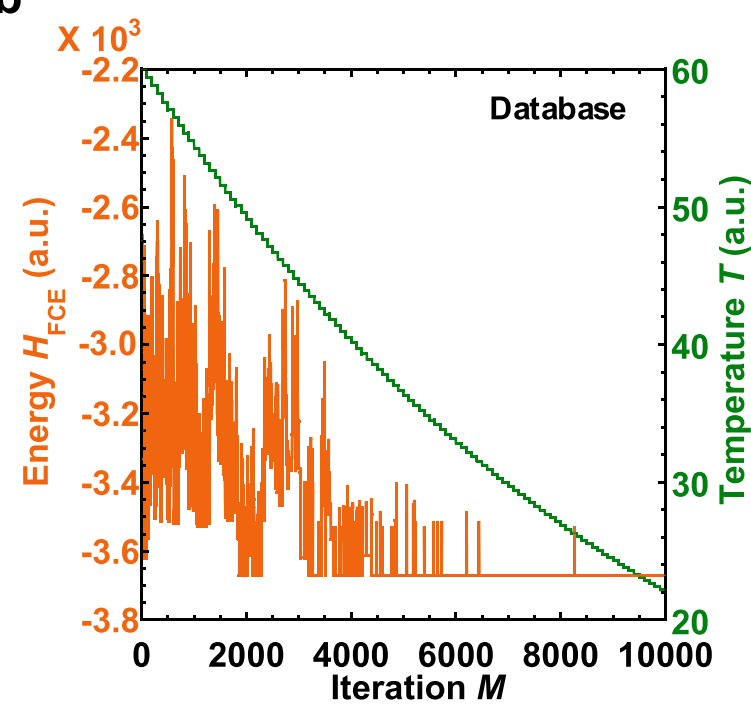

Iteration $M=10,000$ Optimum Solution@ $H_{\mathrm{FCE}}=-3,670$

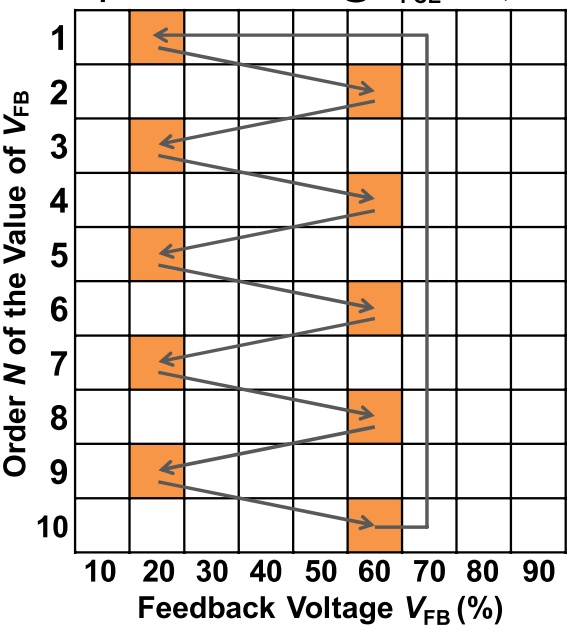

Figure 3. Optimization of FCE schedule using ground-state searches. Energy history of the ground-state search of the Ising spin model (a) for the optimal solution that we provide and specify the preset (blue), and (b) for the experimental data stored in the database (orange). The annealing schedule (green) decreases nonlinearly. Our simulated annealing algorithm uses single spin-flip Metropolis updates with a linear profile of inverse temperatures $\beta=T^{-1}$, where $\beta$ is calculated by $60 \times 0.99$ (with $\beta$ updated every 100 iterative cycles over the lattice spins). The energy profiles of (a,b) exhibit similar characteristics $(A=5, B=430, C=550, D=360$, $\left.h_{\mathrm{i}, \mathrm{x}}=1\right)$. Final spin status after 10,000 iterative cycles for the (c) test problem and (d) database. Spin states in orange and white represent up spin and down spin states, respectively. Here, the optimal solution of our simulation is identified as follows: $V_{\mathrm{FB}}(\%)=60 \rightarrow 70 \rightarrow 80 \rightarrow 90 \rightarrow 10 \rightarrow 20 \rightarrow 30 \rightarrow 40 \rightarrow 50 \rightarrow 60$ in the (c) (test problem) and $V_{\mathrm{FB}}(\%)=20 \rightarrow 60 \rightarrow 20 \rightarrow 60 \rightarrow 20 \rightarrow 60 \rightarrow 20 \rightarrow 60 \rightarrow 20 \rightarrow 60$ in the (d) (database).

$$
\text { Score }_{\text {process }} \equiv S_{N}=\sum_{n=1}^{N} s_{n, n+1}(X, Y) .
$$

Notice that $s_{\mathrm{n}, \mathrm{n}+1}(X, Y)$ is $s_{N, 1}(X, Y)$ when $n=N$. In other words, Eq. (8) evaluates the entire $V_{\mathrm{FB}}$ scheduling. Because $N$ is set to 10 in this study, a $V_{\mathrm{FB}}$ schedule consisting of $10 \mathrm{FBs}$ is evaluated by Eq. (8), and $S_{10}$ is calculated. The detailed calculation procedure of $S_{10}$ is as follows. First, a $V_{\mathrm{FB}}$ schedule consisting of $10 \mathrm{FBs}$ is generated with Ising spin computing or random selection. Next, $s_{1,2}, s_{2,3}, \ldots$, and $s_{10,1}$ are scored by the database shown in Fig. 2c. Finally, $S_{10}$ is calculated by adding $s_{1,2}, s_{2,3}, \ldots$, and $s_{10,1}$. Figure 4 a presents a histogram of the $S$ core $e_{\text {process }}$ obtained from the Ising spin computing (orange histogram) and random selection (blue histogram) when both are performed 1,000 times. All initial spin values were random in all experiments described in this paper to explore the various conditions. The blue histogram in Fig. 4a exhibits uniform Gaussian-like score distributions, 
a

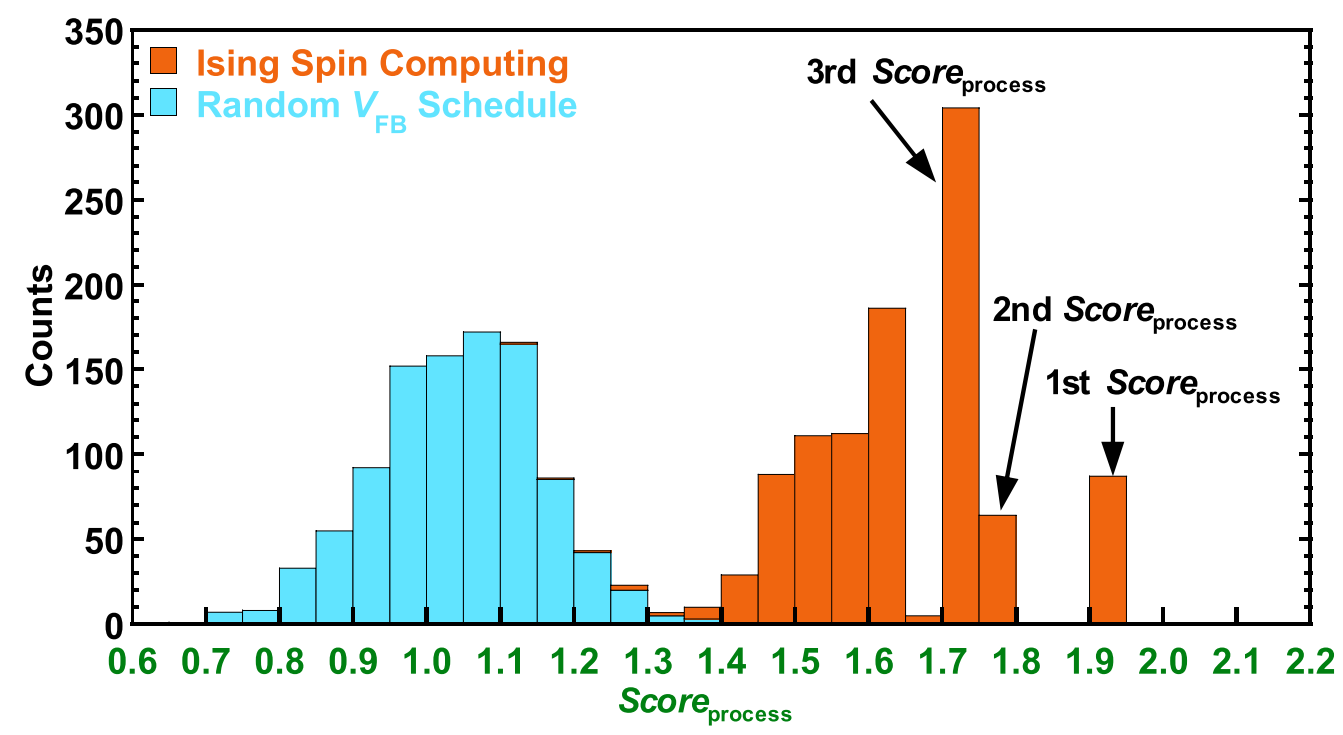

b

C

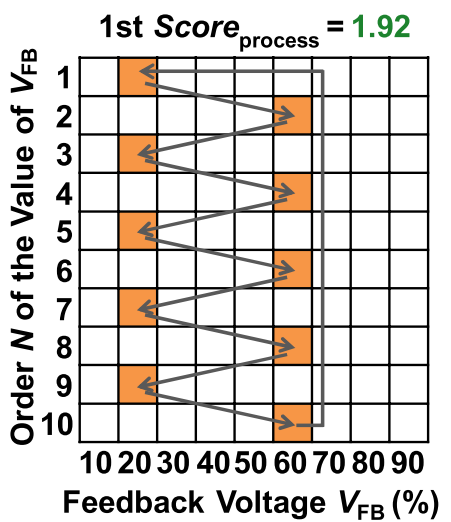

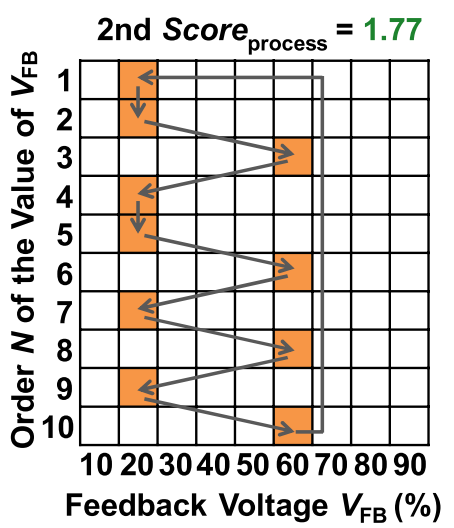

d

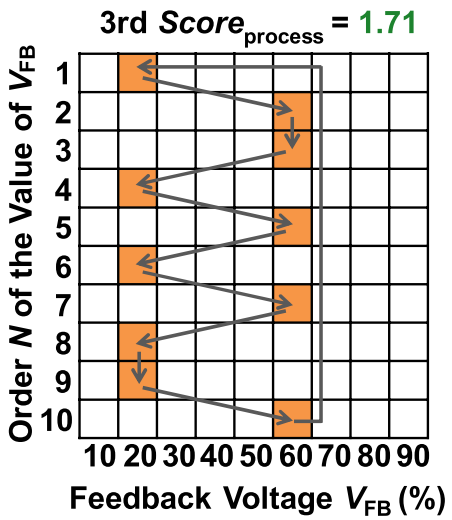

Figure 4. Ability of Ising spin computing to search for the ground-state solution. (a) Number of Ising spin

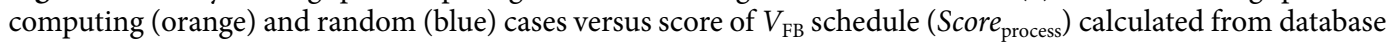
$\left(A=5, B=430, C=550, D=360, h_{\mathrm{i}, \mathrm{x}}=1, \beta=60\right)$. The values of Score $_{\text {process }}$ for Ising spin computing and random cases are obtained by adding 10 times of $S$ core ${ }_{\text {trans }}$ for each $V_{\mathrm{FB}}$ schedule obtained by each method. (b-d) The top three final spin statuses corresponding to each score out of (a). All statuses are the results obtained as output from Ising spin computing, and the highest rated $(\mathbf{b})$ is the optimal $V_{\mathrm{FB}}$ schedule produced from the database.

which represent the validity of the database. In addition, the distribution of the histogram shifting to the right indicates that the highly rated $V_{\mathrm{FB}}$ schedule is selected. From Fig. $4 \mathrm{a}$, the orange histogram of the Ising spin computing is significantly shifted to the right as compared with the histogram obtained from random selection. This result indicates that Ising spin computing can automatically select the highly evaluated $V_{\mathrm{FB}}$ schedules through the ground-state searches of the solutions. Figure $4 \mathrm{~b}-\mathrm{d}$ show the top three final spin statuses corresponding to each Score $_{\text {process. }}$ The highest scoring solution (Fig. $4 \mathrm{~b}$ ) is considered the optimum schedule of $V_{\mathrm{FB}}$. Further, the results of the scoring solutions for the second and third place in Fig. 4c,d indicate that the heuristic solutions almost agree with the optimal schedule. Therefore, the local minimum solutions obtained by Ising spin computing are also considered suitable for use in the FCE method. These results show that Ising spin computing could discover a remarkable amount of information about the FCE by starting from disorderly generated data; that is, these are the results of applying machine learning to disordered data to obtain order in exploring the experimental parameters of the FCE method.

FCE experiments with results of ground-state searches of experimental parameters. Figure 5a,b present the conductance traces recorded during the FCE with the schedule of the 1st Score $e_{\text {process }}$ obtained from the Ising spin computing and random $V_{\mathrm{FB}}$ scheduling, respectively, which are prepared as a 
a
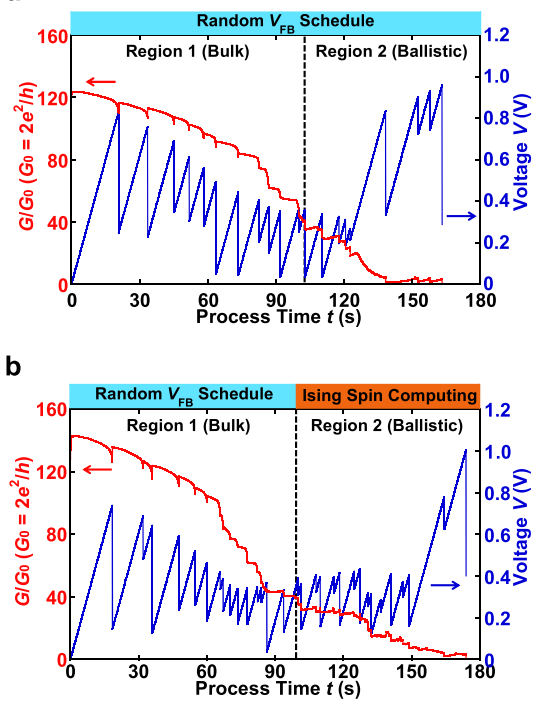

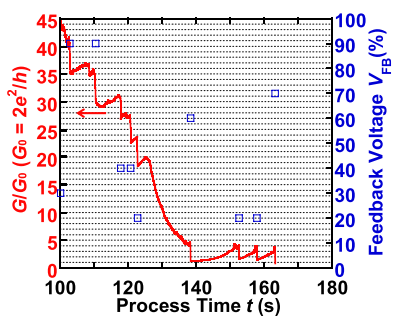

d

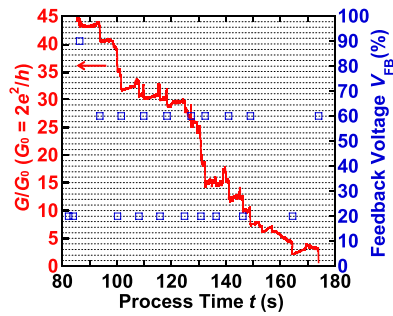

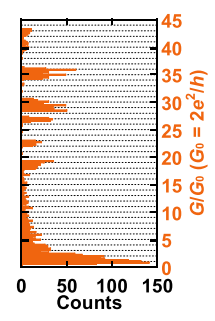

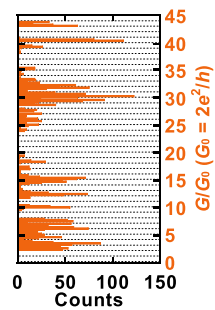

g
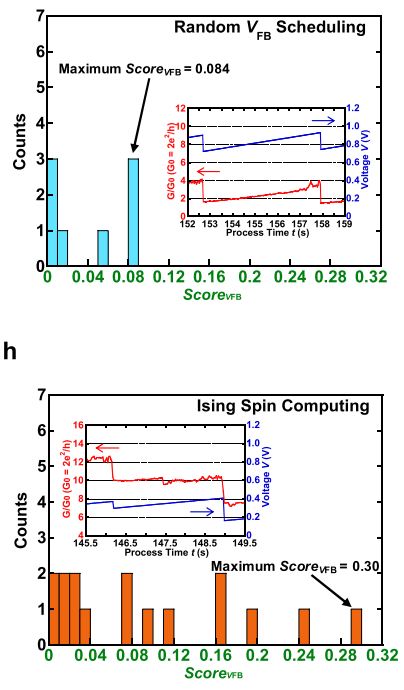

Figure 5. Comparison of Ising spin computing method with random method. (a,b) Time evolution of conductance for Au nanowires during the FCE process. A random $V_{\mathrm{FB}}$ schedule is applied for both methods in the range of $G \geq 40 G_{0}$ (region 1). In the range of $G \leq 40 G_{0}$ (region 2), the random $V_{\mathrm{FB}}$ schedule is applied in (a) and the optimal $V_{\mathrm{FB}}$ schedule obtained by the ground-state searches of the Ising spin model is applied in (b). (c,d) Conductance traces recorded for ballistic regime $\left(G \leq 45 G_{0}\right)$. (e,f) Conductance histograms obtained for $(\mathbf{c}, \mathbf{d})$, respectively. Because of the constant plateaus, the conductance histogram in (f) exhibits sharp peaks at integer multiples of the conductance quantum compared with (e). (g,h) Distributions of the Score $e_{V F B}$ constructed from the region 2 in (a,b), respectively. The insets in $(\mathbf{g}, \mathbf{h})$ are conductance curves showing the largest Score $_{V F B}$.

reference. In the initial stage denoted as region 1 , where the conductance is larger than $40 G_{0}$, the conductance decreases continuously because of the EM of Au atoms. This result indicates that region 1 shows the bulk regime of electrical conduction. However, as the conductance decreases to less than $40 G_{0}$, conductance plateaus and steps at and near integer multiples of $1 G_{0}$ are observed, as seen in region 2. This result implies that region 2 shows the ballistic conduction regime and that EM proceeds with the behavior of the one-by-one removal of single Au atoms ${ }^{34,35}$. Here, to compare both methods from the viewpoint of the controllability of conductance quantization, enlarged views of region 2 are shown in Fig. $5 c$,d. The conductance changes in steps, consisting of plateaus and jumps. Further, Fig. 5e,f present histograms of the conductance values derived from the EM data of Fig. 5c,d, respectively, in the range $G \leq 45 G_{0}$. As the conductance changes whenever the atomic structure changes at the metal contacts, stable structures can yield preferred peaks in the conductance histograms ${ }^{33}$. Therefore, the sharp peaks indicate that particular conductance values and their corresponding contact configurations are more stable. Conductance plateaus and steps at and near integer multiples of $G_{0}$ are observed in Fig. $5 \mathrm{f}$ as compared to Fig. 5e. We further applied Eq. (1) to each conductance trace in the range $G \leq 40 G_{0}$ to compare $V_{\mathrm{FB}}$ scheduling in terms of $S_{c o r e}{ }_{V F B}$. Figure $5 \mathrm{~g}$, h show the $S_{c o r e}{ }_{V F B}$ and the insets show the highest evaluated conductance traces. As shown in Fig. $5 \mathrm{~g}$,h, the results of the optimal $V_{\mathrm{FB}}$ scheduling obtained from Ising spin computing are superior to those from random $V_{\mathrm{FB}}$ scheduling not only from the conductance histograms but also from the viewpoint of $S$ core ${ }_{V \mathrm{FB}}$. In addition, the conductance trace in the inset of Fig. 5h shows conductance plateaus around $10 G_{0}$. These results suggest that the ballistic conduction regime in the electrical conduction is precisely controlled by the $V_{\mathrm{FB}}$ scheduling obtained by the ground-state solution of the Ising spin computing, despite the complicated phenomenon of mass transport in electromigrated nanowires.

To gain insights into the dynamics of EM in Au nanowires, we accumulated $V_{\mathrm{C}}$ and plotted its histogram in Fig. 6a (applied using random $V_{\mathrm{FB}}$ schedule) and Fig. 6b (applied using optimized $V_{\mathrm{FB}}$ schedule). $V_{\mathrm{C}}$ is the maximum value of the junction voltage $V_{\mathrm{J}}$ for each FB cycle ${ }^{34,35}$. Each histogram consists of data collected from 22 samples. In addition, each inset in Fig. 6a,b shows a histogram constructed from the $V_{\mathrm{C}}$ accumulated for the region of $G \geq 40 G_{0}$. Umeno et al. ${ }^{34,35}$ reported that $e V_{\mathrm{C}}$ can be regarded as "the chemical potential for atom migration" and, hence, the obtained histogram can be interpreted as "the spectrum of electromigration". As seen in Fig. 6a,b, major peaks coincide with the activation energies for the surface potential of Au adatoms ${ }^{36-38}$, results that are similar to those in ref. ${ }^{35}$. Hence, Fig. $6 \mathrm{a}, \mathrm{b}$ clearly indicate that the process of EM at the Au nanowires is driven by the microscopic kinetic energy transfer from a single conduction electron to a single metal atom ${ }^{34,35}$. In addition, the difference between Fig. 6a,b appears in the range from 0.26 to $0.4 \mathrm{eV}$, showing a major peak at $0.3-0.32 \mathrm{eV}$ in the case of Fig. $6 \mathrm{~b}$. This value is equal to the activation energy for the self-diffusion on the (100) surfaces, which was predicted by the embedded atom model ${ }^{38}$. This is due to the difference in the $V_{\mathrm{FB}}$ scheduling in the ballistic regime $\left(G \leq 40 G_{0}\right)$ between the random $V_{\mathrm{FB}}$ schedule (Fig. 6a) and the $V_{\mathrm{FB}}$ schedule optimized by Ising spin computing (Fig. 6b). The findings obtained here provide a solid basis for understanding the migration of Au atoms at atomic junctions driven by a controlled EM method. 
a

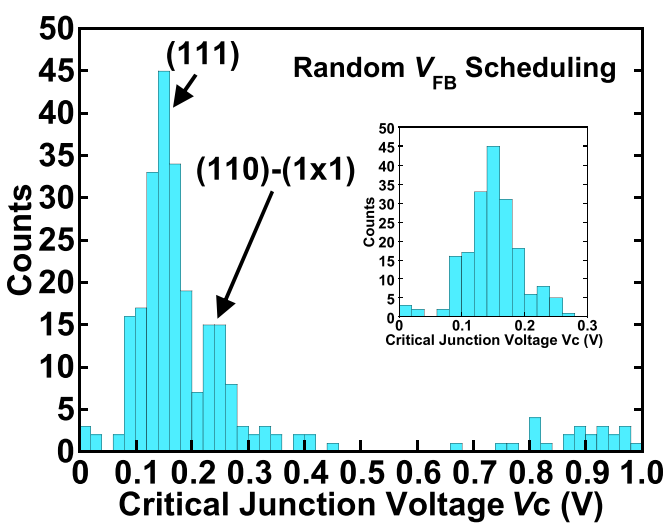

b

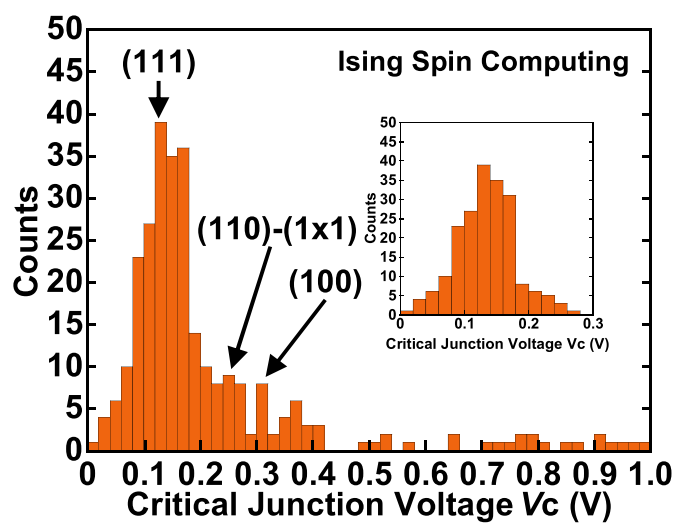

Figure 6. Histogram of the critical values of the junction voltage, $V_{c}$. The histograms are constructed from the results of applying a (a) completely random $V_{\mathrm{FB}}$ schedule and (b) $V_{\mathrm{FB}}$ schedule, where the optimal parameters are applied at $G \leq 40 G_{0}$. The insets in $(\mathbf{a}, \mathbf{b})$ are histograms of the $V_{\mathrm{C}}$ accumulated for the region of $G \geq 40 G_{0}$. The major peaks agree well with the surface self-diffusion potentials of Au atoms. A remarkable finding is that the large peak at $0.12-0.14 \mathrm{eV}$ coincides with the activation energy for self-diffusion on the (111) surfaces ${ }^{36}$. The small peak visible at $0.26-0.28 / 0.24-0.26 \mathrm{eV}$ is close to the self-diffusion on the $(110)-(1 \times 1)$ surfaces predicted by molecular dynamics simulations ${ }^{37}$. Furthermore, $0.3-0.32 \mathrm{eV}$ is close to the theoretically predicted activation energy for the self-diffusion energy on the (100) surfaces $^{38}$.

\section{Conclusions}

In this study, we established a novel design method for determining FCE parameters using Ising spin computing. Optimization of the parameters of the FCE process using the Ising spin computing method provides an alternative to the conventional method with human intervention that requires expert knowledge of the physical processes and methods involved. The advantages of using the Ising spin model over traditional machine-learning methods for small training datasets have been reported ${ }^{27,28}$. Further, we showed that the FCE process can be optimized with a small database of only 50 samples. Therefore, in areas of research where datasets with a small number of relevant samples may be more common, Ising spin computing may be the algorithm of choice. However, this improvement could be even greater when expanding the database and/or optimizing more FCE parameters such as $G_{\mathrm{TH}}$ and $V_{\mathrm{STEP}}$ using Ising spin computing because the database is too small. In fact, as the size of the training dataset increased, the results improved to a certain degree in "a Higgs optimization problem" 27 " and "a simplified computational biology problem 28 ." Furthermore, in the case of maximum-cut problems, the accuracy of the solutions remained almost constant for various problem sizes ${ }^{39}$. Hence, the same tendency is expected to be obtained in FCE parameter optimization with Ising spin computing.

In this study, ground-state searches of Ising spin computing were performed by simulated annealing, which is a classical method. However, in the future, the development of quantum annealers may make it possible to optimize the experimental parameters of FCE using quantum annealing considering that various optimization problems have been solved with commercially available quantum annealers ${ }^{23-30}$. Therefore, the quantum annealing technique is expected to provide appropriate solutions or optimize the experimental parameters of FCE. Furthermore, the Ising spin model features intrinsically nonlocal correlations that can lead to substantially more compact representations of many-body quantum states. Therefore, their effectiveness for the optimization of the experimental parameters of the FCE method makes them a powerful tool for applications beyond the formation of nanogaps and/or controlling quantized conductance in nanowires, including understanding complicated quantum-mechanical systems.

\section{Methods}

Au nanowire fabrication and design. A Si wafer with a thermally grown $\mathrm{SiO}_{2}$ layer is used as the substrate; next, a positive tone resist was spin coated onto the substrate. The electrode pattern with a constriction of $\sim 100 \mathrm{~nm}$ was fabricated using electron-beam lithography. A standard development procedure was applied to remove the resist by inserting the substrate into a development solution, after which the substrate was transferred into propanol to terminate the development process. The resist layer served as a mask for deposition of the metal layer, after which the sample was immersed in acetone for the lift-off process to remove the redundant part of the metal layer.

Electrical characterization. A source-measure unit was used for sourcing the voltage (up to $10 \mathrm{~V}$ and with $50 \mu \mathrm{V}$ sensitivity) and measuring the voltage (up to $10 \mathrm{~V}$ with $10 \mu \mathrm{V}$ sensitivity) and the current (up to $10 \mathrm{~mA}$ with $10 \mathrm{nA}$ sensitivity), allowing the characterization of current-voltage behavior with a time resolution of $25 \mathrm{~ms}$. All measurements were carried out under a two-terminal arrangement. The FCE experiments for the Au nanowires were performed at room temperature under ambient conditions using a real-time operating system (RTOS)-based controller, which is specifically designed to run FCE applications with precise timing and reliability. The system consisted of a host PC, RTOS, and target samples of Au nanowires. In this system, we first 
designed and coded the FCE algorithm on a host PC before deploying the algorithm in the RTOS. As a result, the embedded algorithm with a user-defined program could be performed with precise timing and reliability.

Ising spin computing. $\quad W_{x, y}$ is calculated by

$$
W_{x, y}=100-\frac{\operatorname{avg} \operatorname{Score}_{\text {trans }}(X, Y)-\min \operatorname{Score}_{\text {trans }}(X, Y)}{\max \operatorname{Score}_{\text {trans }}(X, Y)-\min \operatorname{Score}_{\text {trans }}(X, Y)} \times 99,
$$

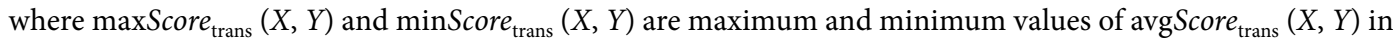
the database, respectively, and avgScore trans $(X, Y)$ represents the average of each $\operatorname{Score}_{\text {trans }}(X, Y)$. By using Eq. (9), when avg $\operatorname{Score}_{\text {trans }}(X, Y)$ is the largest and the smallest, $W_{x, y}$ becomes 1 and 100 , respectively. $W_{x, y}$ is set to 100 in case of no information of $S_{c o r e}$ trans $(X, Y)$. In our formulation of the Hamiltonian, each term $(A, B, C$, and $D)$ is derived heuristically, based on the size of the problem. At the beginning, the values of $C$ and $D$ are set to an initial value such that the number of up spins would be $N$ (with the values of $A$ and $B$ set to 0 ). Then, each row has exactly one up spin by setting the value of $B$. Finally, the values of $A$ are gradually increased within the range where valid solutions are obtained while adjusting the value of $D$. In the test described in the text, ground-state searches were carried out 100 times with the optimal solution set to $H_{\mathrm{FCE}}$ of $-4,175$ to check the performance of our Ising spin computing ( $W_{x, y}$ is set to 1 or 50$)$. As a result, the global minimum $\left(H_{\mathrm{FCE}}=-4,175\right)$ and the optimum solution are obtained at a rate of $95 \%$.

Database and experimental results of FCE. The number of data obtained from 50 samples was 511 FBs $\left(G<100 G_{0}\right)$, and the maximum FB number obtained in one sample was 22 . The ratio of $V_{\mathrm{FB}}=10,20,30$, $40,50,60,70,80$, and $90 \%$ in the database is $14.3,10.8,9.98,11.2,9.59,11.7,13.7,9.00$, and $11.4 \%$, respectively. The design values for length and width of nanowires are 650 and $85 \mathrm{~nm}, 500$ and $50 \mathrm{~nm}$, and 500 and $100 \mathrm{~nm}$. The data in Fig. 5 were obtained from Au nanowires with the length and width of approximately 300 and $100 \mathrm{~nm}$. In our case, the initial resistance $R$ of samples was typically amounted to around 74-190 $\Omega$. This was measured by sweeping the voltage from -7 to $7 \mathrm{mV}$ in steps of $100 \mu \mathrm{V}$. The average value of $S_{c o r e}{ }_{V F B}$ in the database, Fig. $5 \mathrm{~g}, \mathrm{~h}$ is $0.042,0.040$, and 0.096 , respectively.

Received: 26 March 2019; Accepted: 17 October 2019;

Published online: 07 November 2019

\section{References}

1. Bolotin, K. I., Kuemmeth, F., Pasupathy, A. N. \& Ralph, D. C. Metal-nanoparticle single-electron transistors fabricated using electromigration. Appl. Phys. Lett. 84, 3154 (2004).

2. Arzubiaga, L., Golmar, F., Llopis, R., Casanova, F. \& Hueso, L. E. In situ electrical characterization of palladium-based single electron transistors made by electromigration technique. AIP Adv. 4, 117126 (2014).

3. Rattalino, I. et al. A nanogap-array platform for testing the optically modulated conduction of gold-octithiophene-gold junctions for molecular optoelectronics. RSC Adv. 2, 10985-10993 (2012).

4. Perrin, M. L., Burzuri, E. \& van der Zant, H. S. J. Single-molecule transistors. Chem. Soc. Rev. 44, 902-919 (2015).

5. Johnson, S. L., Sundararajan, A., Hunley, D. P. \& Strachan, D. R. Memristive switching of single-component metallic nanowires. Nanotechnology 21, 125204 (2010).

6. Wang, Q. et al. Single-atom switches and single-atom gaps using stretched metal nanowires. ACS Nano 10, 9695-9702 (2016).

7. Schirm, C. et al. E. A current-driven single-atom memory. Nat. Nanotechnol. 8, 645-648 (2013).

8. Jeong, W., Kim, K., Kim, Y., Lee, W. \& Reddy, P. Characterization of nanoscale temperature fields during electromigration of nanowires. Sci. Rep. 4, 4975 (2014).

9. Park, H., Lim, A. K. L., Alivisatos, A. P., Park, J. \& McEuen, P. L. Fabrication of metallic electrodes with nanometer separation by electromigration. Appl. Phys. Lett. 75, 301 (1999).

10. Trouwborst, M. L., van der Molen, S. J. \& van Wees, B. J. The role of Joule heating in the formation of nanogaps by electromigration. J. Appl. Phys. 99, 114316 (2006).

11. Sordan, R., Balasubramanian, K., Burghard, M. \& Kern, K. Coulomb blockade phenomena in electromigration break junctions. Appl. Phys. Lett. 87, 013106 (2005).

12. Esen, G. \& Fuhrer, M. S. Temperature control of electromigration to form gold nanogap junctions. Appl. Phys. Lett. 87, 263101 (2005).

13. Strachan, D. R. et al. Controlled fabrication of nanogaps in ambient environment for molecular electronics. Appl. Phys. Lett. 86, 043109 (2005).

14. Campbell, J. M. \& Knobel, R. G. Feedback-controlled electromigration for the fabrication of point contacts. Appl. Phys. Lett. 102, 023105 (2013).

15. Kanamaru, Y., Ando, M. \& Shirakashi, J. Ultrafast feedback-controlled electromigration using a field-programmable gate array. J. Vac. Sci. Technol. B 33, 02B106 (2015).

16. Brush, S. G. History of the Lenz-Ising model. Rev. Mod. Phys. 39, 883-893 (1967).

17. Lucas, A. Ising formulation of many NP problems. Front. Phys. 2, 5 (2014).

18. Yamaoka, M. et al. A 20k-spin Ising chip to solve combinatorial optimization problems with CMOS annealing. IEEE J. Solid-State Circuits 51, 303-309 (2016).

19. Johnson, M. W. et al. Quantum annealing with manufactured spins. Nature 473, 194-198 (2011).

20. Utsunomiya, S., Takata, K. \& Yamamoto, Y. Mapping of Ising models onto injection-locked laser systems. Opt. Express 19, 18091-18108 (2011).

21. Puri, S., Andersen, C. K., Grimsmo, A. L. \& Blais, A. Quantum annealing with all-to-all connected nonlinear oscillators. Nat. Commun. 8, 15785 (2017).

22. Mizushima, K., Goto, H. \& Sato, R. Large-scale Ising-machines composed of magnetic neurons. Appl. Phys. Lett. 111, 172406 (2017).

23. Wang, C., Chen, H. \& Jonckheere, E. Quantum versus simulated annealing in wireless interference network optimization. Sci. Rep. 6, 25797 (2016).

24. Tran, T. T. et al. A hybrid quantum-classical approach to solving scheduling problems. Proc. 9th Annual Symp. Combinatorial Search (2016).

25. Neukart, F. et al. Traffic flow optimization using a quantum annealer. Front. ICT 4, 29 (2017). 
26. Perdomo-Ortiz, A., Dickson, N., Drew-Brook, M., Rose, G. \& Aspuru-Guzik, A. Finding low-energy conformations of lattice protein models by quantum annealing. Sci. Rep. 2, 571 (2012).

27. Mott, A., Job, J., Vlimant, J.-R., Lidar, D. \& Spiropulu, M. Solving a Higgs optimization problem with quantum annealing for machine learning. Nature 550, 375-379 (2017).

28. Li, R. Y., Felice, R. D., Rohs, R. \& Lidar, D. A. Quantum annealing versus classical machine learning applied to a simplified computational biology problem. npj Quantum Inf. 4, 14 (2018).

29. Rosenberg, G. et al. M. L. Solving the optimal trading trajectory problem using a quantum annealer. IEEE J. Select. Top. Signal Process. 10, 1053 (2016).

30. Boyda, E. et al. Deploying a quantum annealing processor to detect tree cover in aerial imagery of California. PLoS One 12, e0172505 (2017).

31. Kirkpatrick, S., Gelatt, C. D. \& Vecchi, M. P. Optimization by simulated annealing. Science 220, 671-680 (1983).

32. Denchev, V. S. et al. What is the computational value of finite-range tunneling? Phys. Rev. X 6, 031015 (2016).

33. Kurui, Y., Oshima, Y., Okamoto, M. \& Takayanagi, K. Conductance quantization and dequantization in gold nanowires due to multiple reflection at the interface. Phys. Rev. B 79, 165414 (2009).

34. Umeno, A. \& Hirakawa, K. Nonthermal origin of electromigration at gold nanojunctions in the ballistic regime. Appl. Phys. Lett. 94, $162103(2009)$

35. Umeno, A. \& Hirakawa, K. Spectroscopic analysis of electromigration at gold nanojunctions. Physica E 42, 2826-2829 (2010).

36. Agrawal, P. M., Rice, B. M. \& Thompson, D. L. Predicting trends in rate parameters for self-diffusion on FCC metal surfaces. Surf. Sci. 515, 21-35 (2002).

37. Montalenti, F. \& Ferrando, R. Jumps and concerted moves in $\mathrm{Cu}, \mathrm{Ag}$, and $\mathrm{Au}(110)$ adatom self-diffusion. Phys. Rev. B 59, 5881 (1999).

38. Liu, C. L., Cohen, J. M., Adams, J. B. \& Voter, A. F. EAM study of surface self-diffusion of single adatoms of fcc metals Ni, Cu, Al, Ag, $\mathrm{Au}, \mathrm{Pd}$, and Pt. Surf. Sci. 253, 334-344 (1991).

39. Yoshimura, C. et al. Uncertain behaviours of integrated circuits improve computational performance. Sci. Rep. 5, 16213 (2015).

\section{Author contributions}

S.S. and J.S. conceived the idea that led to the research; S.S. and Y.H. fabricated the devices; S.S. and Y.H. performed experiments and electrical measurements; S.S., Y.H. and M.I. implemented the Ising spin computing; and S.S. and J.S. wrote the main paper and Methods section with input from all other authors.

\section{Competing interests}

The authors declare no competing interests.

\section{Additional information}

Correspondence and requests for materials should be addressed to J.-i.S.

Reprints and permissions information is available at www.nature.com/reprints.

Publisher's note Springer Nature remains neutral with regard to jurisdictional claims in published maps and institutional affiliations.

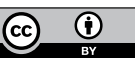

Open Access This article is licensed under a Creative Commons Attribution 4.0 International

License, which permits use, sharing, adaptation, distribution and reproduction in any medium or format, as long as you give appropriate credit to the original author(s) and the source, provide a link to the Creative Commons license, and indicate if changes were made. The images or other third party material in this article are included in the article's Creative Commons license, unless indicated otherwise in a credit line to the material. If material is not included in the article's Creative Commons license and your intended use is not permitted by statutory regulation or exceeds the permitted use, you will need to obtain permission directly from the copyright holder. To view a copy of this license, visit http://creativecommons.org/licenses/by/4.0/.

(C) The Author(s) 2019 